\title{
The spectacular recent trials of urgent neurointervention for acute stroke: fuel for a revolution
}

\author{
How should we redesign our stroke services in light of neurointerventional advances?
}

Stroke

medicine has

come a long

way from the

nihilism of two

decades ago,

with numerous

interventions

now supported

by high-level

evidence

Richard I Lindley $M D, F R C P(E d i n), F R A C P 1,2$

Christopher R Levi BMedSci, MBBS, FRACP 3.4

1 Westmead Clinical School, University of Sydney, Sydney, NSW.

2 The George Institute for Global Health, Sydney, NSW.

3 John Hunter Hospital, Newcastle, NSW.

4 University of Newcastle, Newcastle, NSW.

richard.lindley@ sydney.edu.au

doi: 10.5694/mjal5.00395

Online first 13/7/15 n 2013, neutral results from three trials of neurointervention for treating ischaemic stroke were simultaneously published - a triad of gloom. ${ }^{1-3}$ In just over 2 years since, five positive trials have been reported. ${ }^{4-8}$ What explains this extraordinary turnaround, and what are the implications for stroke services in Australia and around the world? The answers to these questions are surprising and reflect a mixture of science, technology and policy.

\section{The roles of science, technology and policy}

The science involved is the culmination of a decade of work on proving that brain imaging can identify the ischaemic penumbra - the area of the brain that has shut down and is on the path to infarction but, with successful reperfusion, is potentially salvageable. By recruiting patients with a favourable profile for reperfusion therapy (so-called target mismatch, where the ratio of perfusion lesion to established infarct is $>1.8$, the perfusion lesion volume is $>15 \mathrm{~mL}$, and the established infarct volume is $<70 \mathrm{~mL}$ ), ${ }^{9}$ we are now able to identify those who are likely to respond well. In addition, computed tomography (CT) angiography is now widely available and can demonstrate major cerebral vessel occlusion - a clear target for therapy. ${ }^{9}$

In contrast to the neutral trials, the recent trials all used either advanced imaging to identify patients with the "reperfusion responder" profile or angiography to prove major vessel occlusion, or both, then randomly assigned this population of likely responders to receive endovascular reperfusion (usually in addition to alteplase thrombolysis) or standard acute stroke care. The combination therapy resulted in potent reperfusion and a dramatic treatment effect (Appendix), such that three of the five neurointervention trials were stopped early.

The technology is all about the device. In today's fastmoving world, it is almost impossible to design, fund and complete a trial of a device without it becoming obsolete by the time the trial has finished - the fate of the previous studies. ${ }^{1-3}$ Unlike in coronary intervention, the thrombus or embolus in ischaemic stroke must be physically extracted, and the new generation of retrievable stents are a major advance in this regard. One of the recent trials, MR CLEAN, demonstrated that carotid stenting (for extracranial occlusion) was also required for $13 \%$ of the patients receiving intra-arterial treatment. ${ }^{4}$

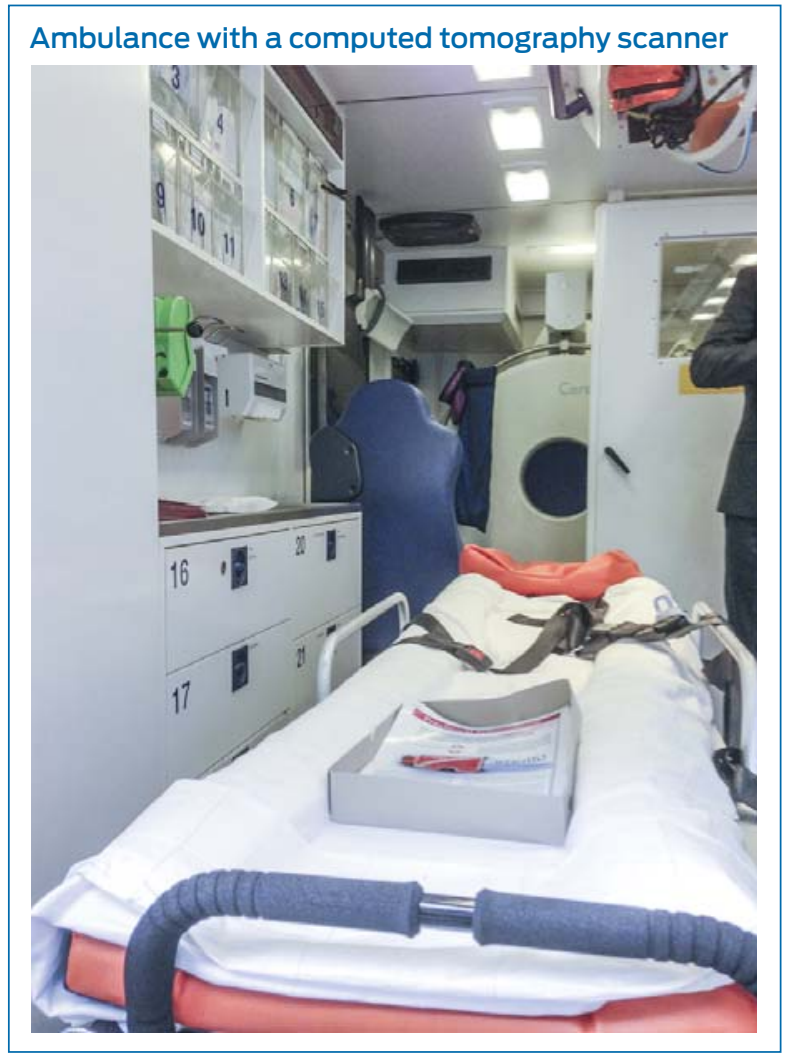

Finally, the unanticipated influence of policy can have profound effects. One of the neutral trials, IMS III, was conducted in the United States at a time when neurointervention was generously compensated. ${ }^{1}$ This, together with the attractiveness of the technology (despite its lack of evidence), meant that most people were treated outside the trial. The difficulties in recruitment (only one or two patients per centre per year) and a possible selection bias of recruiting only "difficult" patients might have had an effect on the results of IMS III.

In contrast, the Dutch MR CLEAN trial provides an important lesson. ${ }^{4}$ All the neurointervention centres in the Netherlands participated in this trial, and from 2013 there was no reimbursement for people treated outside the trial. This allowed some 500 patients to be recruited from 16 sites in just over 3 years, compared with IMS III, which needed 7 years to recruit 656 patients from 58 sites. If trial-only reimbursement for unproven devices were enforced, it is likely that reliable data on efficacy would have been available much earlier, potentially saving hundreds, if not thousands, of lives. 


\section{Implications for practice}

Implementing intravenous thrombolysis has been a difficult and protracted affair, and we are still researching the best ways to achieve it. ${ }^{10}$ However, there has been progress in Australia, thanks to the hard work of clinicians in the state stroke networks. For example, in New South Wales, the Agency for Clinical Innovation led the establishment of a state-wide stroke reperfusion strategy that involved training paramedics to screen for potential thrombolysis candidates with the FAST (Face, Arms, Speech, Time) test and fast-tracking potentially eligible patients to 24-hour thrombolysis centres.

Our challenge is how to redesign our stroke services and how best to build capacity in the neurointerventional workforce. What is the required infrastructure, support and training in advanced imaging selection that would work in our hospitals? The London model of hyperacute stroke centres might work in our capital cities, but the "drip and ship" model of starting thrombolysis followed by urgent transfer to a comprehensive stroke centre may be a solution for outer metropolitan and regional thrombolysis centres. It is abundantly clear that we will never be able to provide on-site neurointervention at all stroke thrombolysis-capable hospitals, nor achieve complete equity of access to endovascular therapy for stroke patients from rural and remote communities.

A potential solution to the shortage of neurointerventionalists is the emerging model to train neurologists in interventional neuroradiological skills. Given there is broad acceptance that a stroke physician (with appropriate training) does not necessarily have to be a neurologist, there is growing support in the US and Australia for the position that a neurointerventionalist does not have to be a radiologist, provided he or she has had appropriate training. ${ }^{11}$

The exact shape of future neurointerventional stroke centres is still uncertain, but the endovascular revolution has arrived, and the stroke community needs to work quickly to redesign stroke care services and build the workforce of specialists trained in endovascular therapies. Drivers for change will include the new national stroke care standard (launched on 10 June 2015) ${ }^{12}$ and the next revision of the national Clinical guidelines for stroke management.

Stroke medicine has come a long way from the nihilism of two decades ago, with numerous interventions now supported by high-level evidence. Immediate brain imaging will identify strokes that are due to haemorrhage, and rapid blood pressure lowering and stroke unit (or intensive) care are the mainstay of treatment for these patients, with surgery needed only for a select few. For patients with ischaemic stroke, revascularisation with appropriate intravenous thrombolysis should be sought, followed by advanced brain imaging to identify patients suitable for additional endovascular therapy.

What is the future? Colleagues in the US and Germany are exploring the utility of an ambulance with an onboard CT scanner (Box), with anecdotal reports of excellent responses to alteplase when given within minutes of major stroke onset. The search is also on for more effective intravenous thrombolytic drugs, with Australia leading an international trial of tenecteplase versus the current standard, alteplase.$^{13}$ However, none of this will be effective without further public health interventions to improve awareness of stroke and the importance of immediately calling 000 for any suspected stroke patient. When it comes to stroke, time is brain.

Competing interests: Richard Lindley has received honoraria from Boehringer Ingelheim (who market alteplase) in his role as a scientific committee member, from Covidien (who market the Solitaire thrombectomy device) for giving two lectures, and from Pfizer. Christopher Levi has received honoraria from Boehringer Ingelheim, Pfizer and BristolMyers Squibb.

Provenance: Commissioned; externally peer reviewed.

References are available online at www.mja.com.au. 
1 Broderick JP, Palesch YY, Demchuk AM, et al; Interventional Management of Stroke (IMS) III Investigators. Endovascular therapy after intravenous t-PA versus t-PA alone for stroke. [Erratum in N Engl J Med 2013; 368: 1265.] N Engl J Med 2013; 368: 893-903.

2 Ciccone A, Valvassori L, Nichelatti M, et al; SYNTHESIS Expansion Investigators. Endovascular treatment for acute ischemic stroke. N Engl J Med 2013; 368: 904-913.

3 Kidwell CS, Jahan R, Gornbein J, et al; MR RESCUE Investigators. A trial of imaging selection and endovascular treatment for ischemic stroke. N Engl J Med 2013; 368: 914-923.

4 Berkhemer OA, Fransen PSS, Beumer D, et al; MR CLEAN Investigators. A randomized trial of intraarterial treatment for acute ischemic stroke. N Engl J Med 2015; 372: 11-20.

5 Campbell BCV, Mitchell PJ, Kleinig TJ, et al; EXTEND-IA Investigators. Endovascular therapy for ischemic stroke with perfusion-imaging selection. N Engl J Med 2015; 372: 1009-1018.

6 Goyal M, Demchuk AM, Menon BK, et al; ESCAPE Trial Investigators. Randomized assessment of rapid endovascular treatment of ischemic stroke. N Engl J Med 2015; 372: 1019-1030.

7 Saver JL, Goyal M, Bonafe A, et al; SWIFT PRIME Investigators. Stent-retriever thrombectomy after intravenous t-PA vs. t-PA alone in stroke. N Engl J Med 2015; 372: 2285-2295.
8 Jovin TG, Chamorro A, Cobo E, et al; REVASCAT Trial Investigators. Thrombectomy within 8 hours after symptom onset in ischemic stroke. N Engl J Med 2015; 372: 2296-2306.

9 Bivard A, Levi C, Krishnamurthy V, et al. Perfusion computed tomography to assist decision making for stroke thrombolysis. Brain 2015; Mar 25: pii: awv071. [Epub ahead of print.]

10 Paul CL, Levi CR, D’Este CA, et al. Thrombolysis ImPlementation in Stroke (TIPS): evaluating the effectiveness of a strategy to increase the adoption of best evidence practice - protocol for a cluster randomised controlled trial in acute stroke care. Implement Sci 2014; 9: 38.

1 Yavagal D; Society of Vascular and Interventional Neurology. About SVIN. SVIN: a neurological society with a new mission. http://www.svin.org/i4a/pages/index.cfm?pageid=3323 (accessed Jun 2015).

12 Australian Commission on Safety and Quality in Health Care. Acute Stroke Clinical Care Standard. Sydney: ACSQHC, 2015. http://www.safetyandquality.gov.au/publications/acutestroke-clinical-care-standard (accessed Jun 2015).

13 Parsons M, Spratt N, Bivard A, et al. A randomized trial of tenecteplase versus alteplase for acute ischemic stroke. $N$ Engl J Med 2012; 366: 1099-1107. 